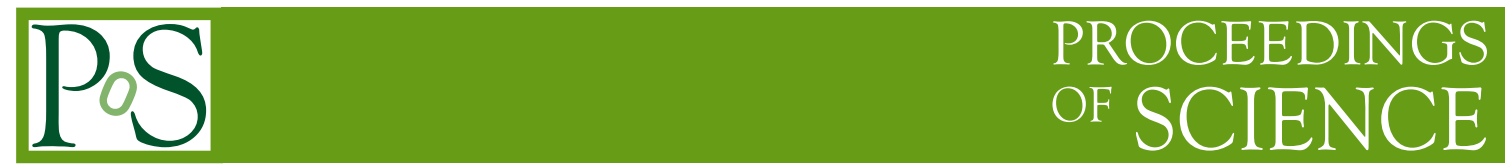

\title{
Velocity Distributions in a Cooling Granular Gas
}

\author{
Sanjay Puri* \\ School of Physical Sciences, Jawaharlal Nehru University, New Delhi - 110067, India \\ E-mail: puri@mail.jnu.ac.in
}

\section{Syed Rashid Ahmad}

School of Physical Sciences, Jawaharlal Nehru University, New Delhi - 110067, India

E-mail: catchrashid@hotmail.com

\begin{abstract}
We use event-driven molecular dynamics simulations to study a freely-evolving granular gas. The system initially loses energy (or cools) in a homogeneous cooling state (HCS). However, fluctuations in the velocity and density fields grow with time and make the system inhomogeneous. This asymptotic state is referred to as the inhomogeneous cooling state (ICS). In the HCS, the scaled velocity distributions deviate from the Maxwell-Boltzmann (MB) function. However, they revert to the MB form in the ICS.
\end{abstract}

International Conference on Statistical Mechanics of Plasticity and Related Instabilities 31 August - 2 September 2005

Bangalore, India

${ }^{*}$ Speaker. 


\section{Introduction}

There has been much research interest in the static and dynamical properties of granular materials or powders $[1,2,3,4,5,6]$. These materials exhibit properties which are intermediate to those of fluids and solids. The understanding and characterization of these properties poses both scientific and technological challenges. Perhaps the most important feature of granular materials is that the grains undergo inelastic collisions, with the normal component of velocity being dissipated on collision. This suggests two classes of dynamical problems in the context of powders:

1) First, let us consider systems where the energy dissipation is compensated by the input of energy from external driving. Then the system settles into a nonequilibrium steady-state, which is often characterized by complex pattern dynamics. There are various standard geometries for agitating granular materials, e.g., rotation in a drum [7, 8], vibration on a platform [9], etc. These systems give rise to diverse examples of pattern formation, which have attracted much research attention.

2) Next, let us consider the evolution of an energized powder in the absence of an external drive. The best-known problem in this class is the cooling of an initially homogeneous system of inelastic particles. The inelastic collisions between particles result in the loss of kinetic energy (or cooling), and the local parallelization of particle velocities. In the early stages, the density field is uniform and the system loses energy in a homogeneous cooling state (HCS) [10]. However, the HCS is unstable to fluctuations in the density and velocity fields, and the system evolves into an inhomogeneous cooling state (ICS) [11, 12, 13, 14, 15, 16, 17, 18]. The ICS is characterized by the emergence and growth of particle-rich clusters, with particles in a cluster moving in approximately parallel directions.

In this paper, we use large-scale molecular dynamics (MD) simulations to study the HCS and ICS of an inelastic granular gas. In earlier work [17], we have characterized pattern formation in the ICS via physical quantities like (a) the correlation function and structure factor for the density and velocity fields; and (b) the growth laws for fluctuations in these fields. Here, we focus on the velocity distributions in the HCS and ICS (for cases with dimensionality $d=2,3$ ). For an elastic hard-sphere gas, an arbitrary velocity distribution rapidly evolves to the Maxwell-Boltzmann (MB) distribution. The situation is not so simple for granular materials with non-MB distributions (e.g., power laws, stretched exponentials, etc.) being reported in various studies $[1,2,3,4,5,6]$.

This paper is organized as follows. In Sec. 2, we briefly review our understanding of the cooling problem. In Sec. 3, we present numerical results from our MD simulations. In Sec. 4, we conclude this paper with a summary and discussion.

\section{Phenomenology of the Cooling Problem}

Let us consider a homogeneous granular gas, comprised of identical hard spheres with mass $m=1$ and diameter $\sigma=1$. Consider a collision between particles labeled $i$ and $j$. The postcollision velocities of the particles $\left(\vec{v}_{i}^{\prime}\right.$ and $\left.\vec{v}_{j}^{\prime}\right)$ are determined from the pre-collision velocities $\left(\vec{v}_{i}\right.$ and $\vec{v}_{j}$ ) by the following rule:

$$
\begin{aligned}
& \vec{v}_{i}^{\prime}=\vec{v}_{i}-\frac{1+e}{2}\left[\hat{n} \cdot\left(\vec{v}_{i}-\vec{v}_{j}\right)\right] \hat{n}, \\
& \vec{v}_{j}^{\prime}=\vec{v}_{j}+\frac{1+e}{2}\left[\hat{n} \cdot\left(\vec{v}_{i}-\vec{v}_{j}\right)\right] \hat{n}
\end{aligned}
$$


where $e(<1)$ is the restitution coefficient. Here, $\hat{n}$ is a unit vector parallel to the relative position of the particles, and points from $j$ to $i$ at the time of collision.

In analogy with the ideal gas, we define the "granular temperature" as the average kinetic energy:

$$
T=\frac{\left\langle\vec{v}^{2}\right\rangle}{d}
$$

where $\left\langle\vec{v}^{2}\right\rangle$ is the mean-squared velocity. The homogeneous state cools with time $t$ as [10]

$$
\frac{d T}{d t}=-\frac{\varepsilon \omega(T) T}{d}, \quad \varepsilon=1-e^{2},
$$

where $\omega(T)$ is the collision frequency at temperature $T$. From kinetic theory, this has the approximate form [19]:

$$
\omega(T) \simeq \frac{2 \pi^{(d-1) / 2}}{\Gamma(d / 2)} \chi(n) n T^{1 / 2},
$$

where $\chi(n)$ is the pair correlation function at contact for hard spheres with density $n$. Substituting the expression for $\omega(T)$ in Eq. (2.3) and integrating, we obtain Haff's cooling law for the HCS:

$$
T(t)=T_{0}\left[1+\frac{\varepsilon \omega\left(T_{0}\right)}{2 d} t\right]^{-2},
$$

where $T_{0}$ is the initial temperature. Notice that the collision time-scale becomes slower due to the ongoing cooling of the granular gas. Therefore, it is convenient to introduce another measure of time, i.e., the average number of collisions $\tau(t)$ that a particle suffers till time $t$ :

$$
\tau(t)=\int_{0}^{t} d t^{\prime} \omega\left(t^{\prime}\right)=\frac{2 d}{\varepsilon} \ln \left[1+\frac{\varepsilon \omega\left(T_{0}\right)}{2 d} t\right] .
$$

The collision time $\tau$ grows logarithmically with real time $t$ for $\varepsilon>0(e<1)$. In the elastic limit $\varepsilon \rightarrow 0(e \rightarrow 1)$, Eq. (2.6) reduces to the expected form, $\tau(t)=\omega\left(T_{0}\right) t$. In terms of the collision time, Haff's cooling law simplifies as

$$
T(\tau)=T_{0} \exp (-\varepsilon \tau / d)
$$

Next, let us discuss velocity distribution functions in the HCS. The natural framework to study these is the inelastic version of the Boltzmann transport equation, which is applicable when velocity correlations are negligible [6]. The elastic case $(e=1)$ is characterized by the MB distribution:

$$
P_{\mathrm{MB}}(\vec{v})=\left(\frac{1}{\pi v_{0}^{2}}\right)^{d / 2} \exp \left(-\frac{\vec{v}^{2}}{v_{0}^{2}}\right), \quad v_{0}^{2}=\frac{2\left\langle\vec{v}^{2}\right\rangle}{d} .
$$

In the near-elastic case $(e \simeq 1)$, the distribution function is time-dependent due to the cooling process, but has a scaling form which differs from the MB function [20, 21]:

$$
P(\vec{v}, t)=\frac{1}{v_{0}^{d}(t)} F\left[\frac{\vec{v}}{v_{0}(t)}\right] \equiv \frac{1}{v_{0}^{d}(t)} F(\vec{c}) .
$$


Here, $v_{0}^{2}(t)=2\left\langle\vec{v}^{2}\right\rangle / d$ as before, and

$$
F(\vec{c})=\frac{1}{\pi^{d / 2}} \exp \left(-c^{2}\right) \sum_{n=0}^{\infty} a_{n} S_{n}\left(c^{2}\right)
$$

In Eq. (2.10), $F(\vec{c})$ has been expanded in terms of the orthogonal Sonine polynomials, which satisfy

$$
\int_{0}^{\infty} d c c^{d-1} \exp \left(-c^{2}\right) S_{n}\left(c^{2}\right) S_{m}\left(c^{2}\right)=\delta_{n m} \frac{\Gamma(n+d / 2)}{2 n !} .
$$

The first few Sonine polynomials are

$$
\begin{aligned}
& S_{0}\left(c^{2}\right)=1 \\
& S_{1}\left(c^{2}\right)=\frac{d}{2}-c^{2}, \\
& S_{2}\left(c^{2}\right)=\frac{d(d+2)}{8}-\frac{(d+2)}{2} c^{2}+\frac{1}{2} c^{4}, \quad \text { etc. }
\end{aligned}
$$

The MB function in Eq. (2.8) arises on setting $a_{0}=1$ and $a_{n}=0(n \geq 1)$ in Eq. (2.10).

The coefficients $a_{n}$ in Eq. (2.10) quantify the deviation from the MB function. The normalization condition dictates $a_{0}=1$, and a scaling argument yields $a_{1}=0$. The first nontrivial coefficient is $a_{2}$ and this has been calculated from kinetic theory as [21]

$$
a_{2}=\frac{16(1-e)\left(1-2 e^{2}\right)}{9+24 d+8 d e-41 e+30(1-e) e^{2}} .
$$

Brey et al. [22] have confirmed the result in Eq. (2.13) via Monte Carlo (MC) simulations of the inelastic Boltzmann equation. However, it is more relevant to study the applicability of these results directly in a cooling granular gas. In this context, Huthmann et al. [23] have undertaken MD simulations of inelastic hard disks in $d=2$. The initial condition for their simulations was a homogeneous state with an MB velocity distribution. Huthmann et al. studied the evolution of $a_{2}$ from its initial value $a_{2}=0$ (the MB value). To obtain the coefficients $a_{n}(t)$, we use the expansion:

$$
\begin{aligned}
\left\langle c^{2 k}\right\rangle(t) & =\left\langle c^{2 k}\right\rangle_{\mathrm{MB}} \sum_{n=0}^{k}(-1)^{n} \frac{k !}{(k-n) ! n !} a_{n}(t), \\
\left\langle c^{2 k}\right\rangle_{\mathrm{MB}} & =\frac{\Gamma(k+d / 2)}{\Gamma(d / 2)} .
\end{aligned}
$$

The first few $a_{n}$ 's are obtained as follows:

$$
\begin{aligned}
& a_{1}(t)=1-\frac{\left\langle c^{2}\right\rangle}{\left\langle c^{2}\right\rangle_{\mathrm{MB}}}=0, \\
& a_{2}(t)=-1+\frac{\left\langle c^{4}\right\rangle}{\left\langle c^{4}\right\rangle_{\mathrm{MB}}}, \\
& a_{3}(t)=1+3 a_{2}-\frac{\left\langle c^{6}\right\rangle}{\left\langle c^{6}\right\rangle_{\mathrm{MB}}}, \quad \text { etc. }
\end{aligned}
$$

Huthmann et al. found that $a_{2}(t)$ evolved to a "steady-state" value consistent with Eq. (2.13). However, the kinetic-theory prediction was not valid in the ICS, and $a_{2}$ relaxed back to zero on a 
time-scale $\tau \sim 30$ collisions for $e=0.4 \ll 1$ (see Fig. 9 of Ref. [23]). Similar results were obtained by Nakanishi [18], who found that the "steady-state" was transient even for values of $e \simeq 1$. This is due to the build-up of correlations prior to the onset of the ICS. However, these authors did not investigate velocity distributions deep into the ICS, which is one of the primary aims of the present study.

\section{Results from Molecular Dynamics Simulations}

\subsection{Details of Simulations}

We used event-driven MD to simulate the granular gas in $d=2,3[24,25]$. The translational motion of inelastic particles obeyed the collision rule in Eq. (2.1). The rotational motion of the particles was neglected. The granular gas consisted of $N=10^{6}$ particles confined in a 2- $d$ or 3- $d$ box with periodic boundary conditions. The box sizes were chosen so that the number fraction was $\rho=0.2$ in both cases, with packing fraction $\phi \simeq 0.157$ in $d=2$ and $\phi \simeq 0.105$ in $d=3$.

The system is initialized by randomly placing particles in a box. All of these particles have the same speed but the velocity vector points in random directions so that $\sum_{i} \vec{v}_{i}=0$. This system is relaxed to an $\mathrm{MB}$ velocity distribution by allowing it to evolve till $\tau \sim 100$ with $e=1$, i.e., the elastic limit. This serves as a typical initial condition for our simulation of inelastic hard spheres.

We will subsequently present results for the time-dependence of the temperature and velocity distribution functions. We have obtained results for cases with $e=0.7,0.8,0.9,0.95$. All statistical results presented in this paper correspond to averages over 50 independent initial conditions.

\subsection{Evolution Pictures and Haff's Cooling Law}

In Fig. 1, we show evolution pictures of the density and velocity fields for a $d=2$ granular gas with restitution coefficient $e=0.8$. The velocity field (frames on right) becomes unstable on a faster time-scale than the density field. Its evolution is characterized by the annealing of point defects, consisting of vortices in $d=2$ (and monopoles in $d=3$ ). The evolution of the velocity field in Fig. 1 is analogous to ordering dynamics in the two-component XY model [26]. In the XY model, vortices and anti-vortices annihilate as this reduces the surface tension. However, in the granular gas, the "surface tension" has a dynamical origin, i.e., the local parallelization of particle velocities on collision. Notice that the velocity field is a conserved variable as the particle collisions conserve momentum.

The pattern dynamics in the velocity field is accompanied by a clustering instability in the density field (frames on left of Fig. 1). The formation of clusters can be understood as follows. Consider a density fluctuation in the HCS. In the denser regions, there is more rapid collision of particles and faster cooling. Thus the pressure becomes lower in the denser regions, and particles are sucked into these regions, reinforcing the density fluctuation [11]. The evolution of the granular gas in the ICS is described by nonlinear hydrodynamic equations for the density and velocity fields. In recent work, Das and Puri (DP) [17] have studied pattern dynamics in the nonlinear ICS regime. They characterized pattern formation via growth laws, and the scaling behavior of the correlation functions and structure factors. DP argued that the streaming-and-aggregation dynamics of the granular gas results in conservation on the cluster length scale, which diverges with time. Thus, 

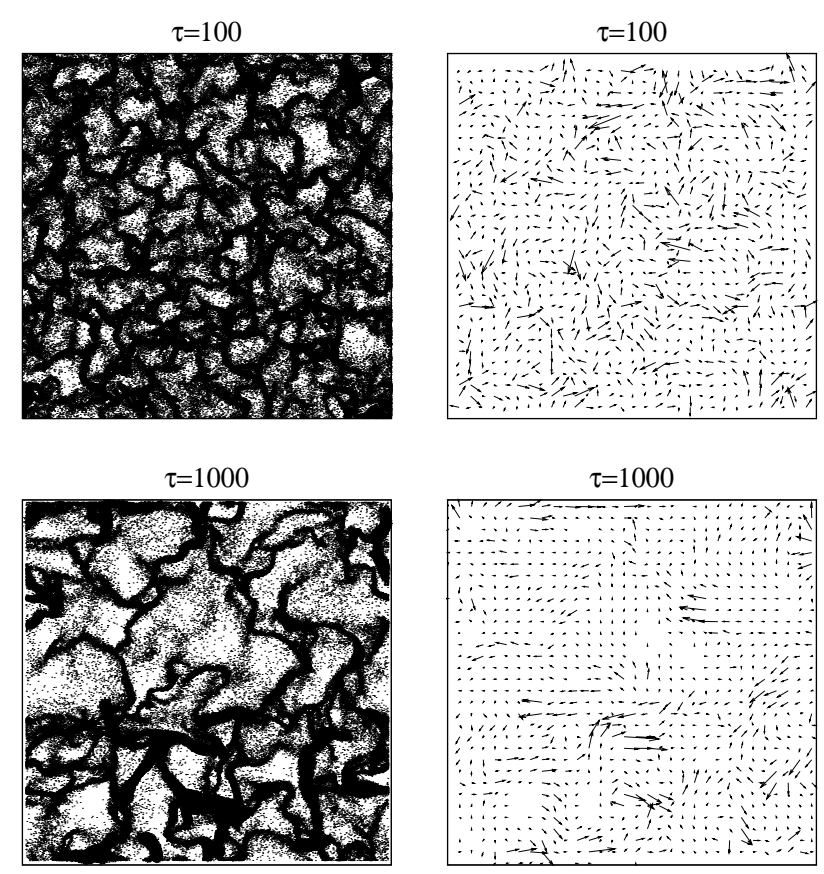

Figure 1: Evolution pictures of the density field (frames on left) and velocity field (frames on right) in the inhomogeneous cooling state of a $d=2$ granular gas. The pictures correspond to $\tau=100,1000$ for a system with particle number $N=262144$, packing fraction $\phi \simeq 0.157$, and restitution coefficient $e=0.8$. The velocity field is obtained by coarse-graining the system into boxes of size $(17.9 \sigma)^{2}$, and plotting the overall velocity for each box.

the asymptotic dynamics obeys a global conservation law, which is a much weaker constraint than a local conservation law. In related work, Wakou et al. [27] have demonstrated that the evolution of the granular flow field can be formulated as a time-dependent Ginzburg-Landau equation for a nonconserved order parameter.

In Fig. 2, we plot the normalized kinetic energy $E(\tau) / E(0)$ vs. $\tau$ for the 2- $d$ case with $e=$ $0.7,0.8,0.9,0.95$. The data is plotted on a linear-log scale - the initial linear decay corresponds to Haff's cooling law for the HCS. The data deviates from Haff's law when correlations build up in the system. We define the HCS $\rightarrow$ ICS crossover time $\tau_{c}$ as the time where the temperature deviates from the Haff prediction by more than ten percent.

In Fig. 3, we show evolution pictures of the density and velocity fields for a $d=3$ granular gas with $e=0.9$. The corresponding $\tau$-dependence of the normalized kinetic energy, $E(\tau) / E(0)$ vs. $\tau$, is shown in Fig. 4.

\subsection{Velocity Distributions in the HCS and ICS}

Next, let us study the velocity distributions in the HCS and ICS. A scaling plot of $F(c)$ vs. $c$ [cf. Eq. (2.9)] does not show significant deviations from the MB function, and we do not show it here. Rather, we directly study the time-dependence of the Sonine coefficients in Eqs. (2.15)(2.17). As the initial velocity distribution is of the MB form, $a_{n}(\tau=0)=0 \forall n>1$. Let us first discuss the behavior of the Sonine coefficient $a_{2}$. In Fig. 5, we plot $a_{2}(\tau)$ vs. $\tau$ for $d=2, e=0.8$; 


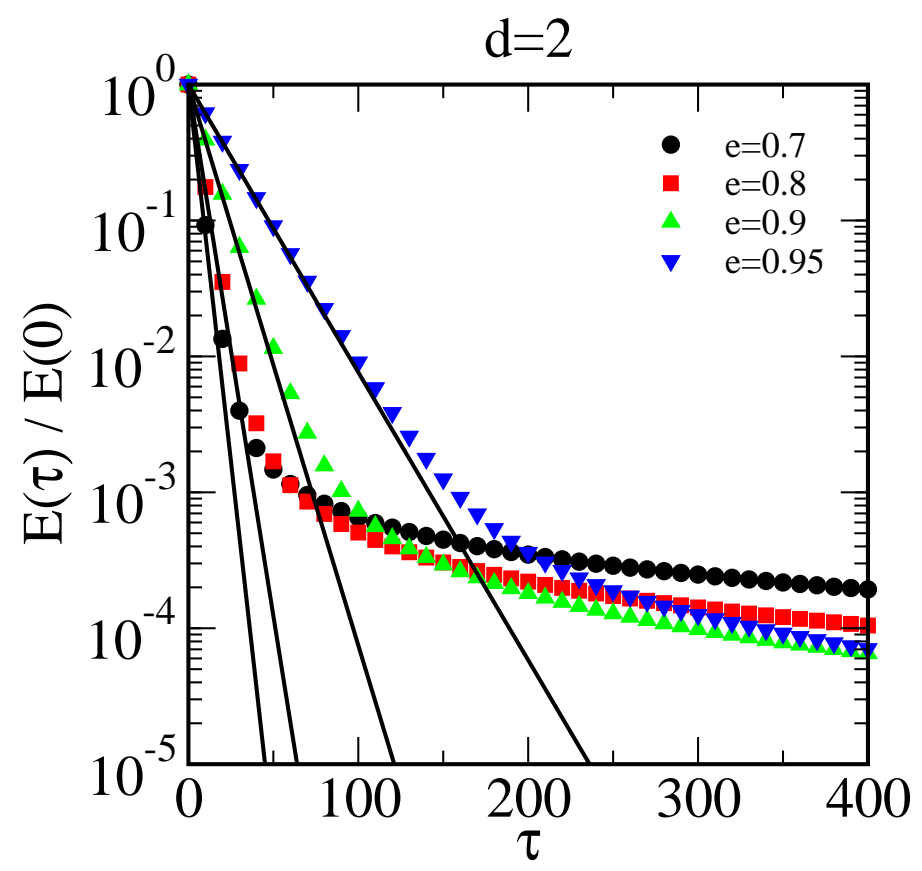

Figure 2: Time-dependence of the granular temperature in $d=2$, shown on a linear-log scale. We plot the normalized kinetic energy $E(\tau) / E(0)$ vs. collision time $\tau$ for $e=0.7,0.8,0.9,0.95$. The solid lines denote Haff's law from Eq. (2.7).
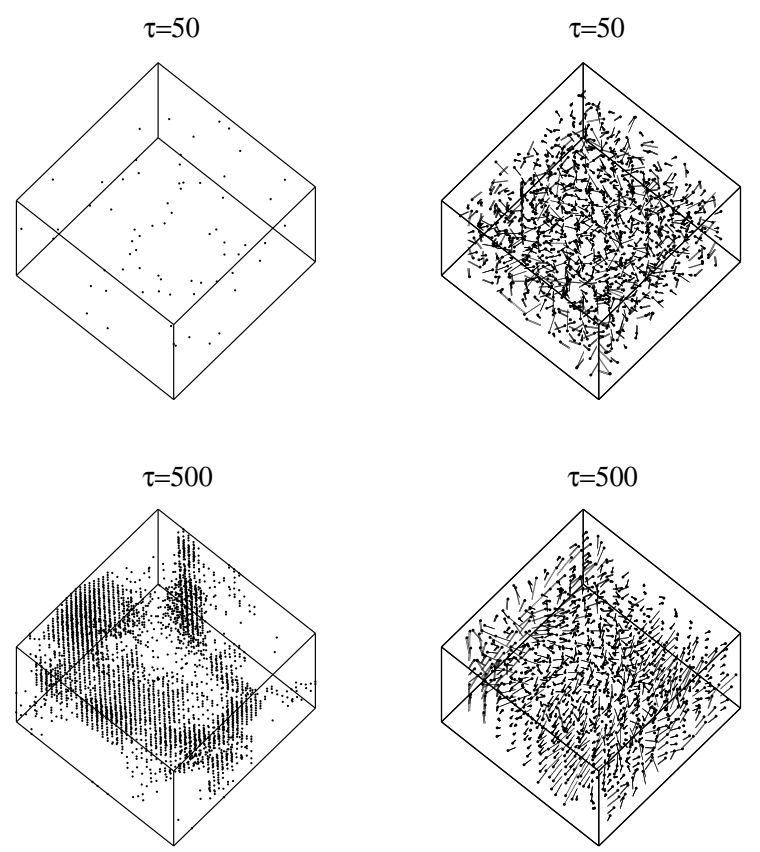

Figure 3: Analogous to Fig. 1 but for the $d=3$ case. The pictures correspond to $\tau=50,500$ for a system with $N=262144, \phi \simeq 0.105$, and $e=0.9$. The density field (frames on left) is plotted by coarse-graining the system into boxes of size $(3.4 \sigma)^{3}$. Boxes with more than 15 particles are marked black, and other boxes are unmarked. For the velocity field (frames on right), we coarse-grain the system into boxes of size $(10.9 \sigma)^{3}$, and plot the overall velocity for each box. 


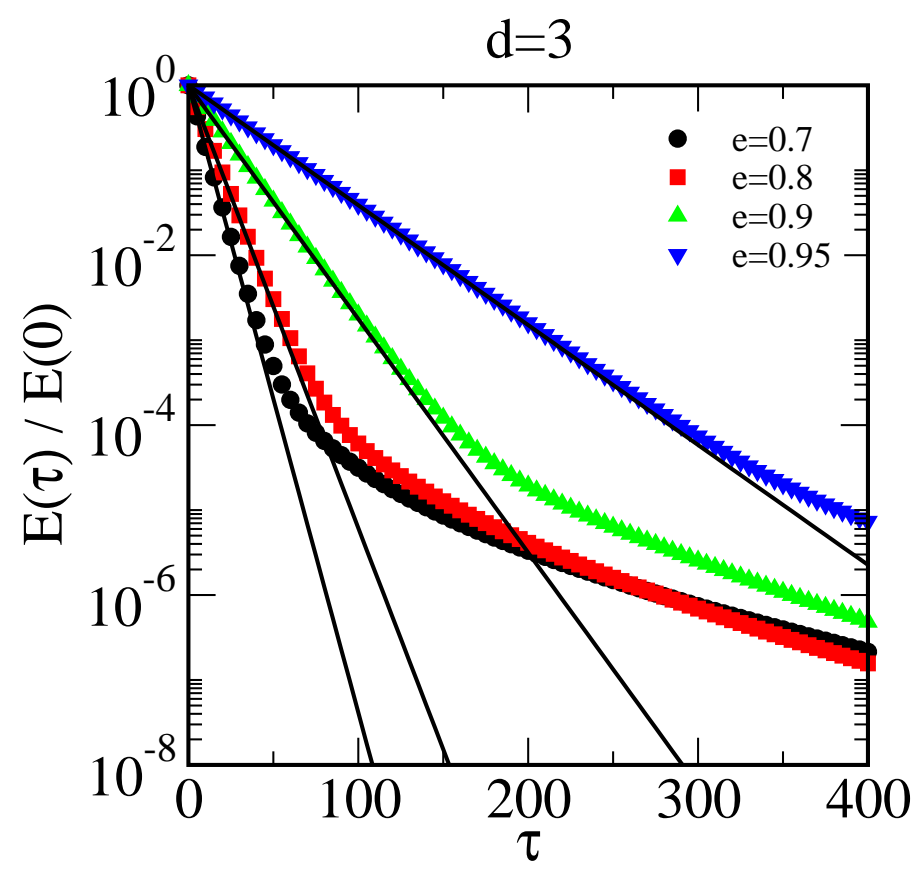

Figure 4: Analogous to Fig. 2, but for the $d=3$ case.

and $d=3, e=0.9$. We show data for individual runs (dotted lines) and their average (solid line). The frames on the left show the early-time behavior, and the frames on the right show the behavior for extended times - upto $\tau=1000$ for $d=2$ and $\tau=500$ for $d=3$. In the early HCS $\left(\tau \ll \tau_{c}\right.$ ), the velocity correlations are negligible and the predictions of kinetic theory should apply. On a time-scale of $\tau \sim 10$ collisions, $a_{2}$ saturates to a "steady-state" value. The data sets in (c) are consistent with the kinetic-theory prediction from Eq. (2.13) - denoted by a horizontal line in the frames on the left. On the other hand, the data set in (a) (for $d=2, e=0.8$ ) does not saturate to the value predicted by Eq. (2.13). Recall that kinetic theory is only applicable when there are no velocity correlations - however, these build up rapidly for $e \ll 1$ and low dimensionality. The frames on the left also show that the data sets for individual runs are comparable in the early timeregime. However, at later times $\left(\tau \gg \tau_{c}\right)$, the individual data sets show strong fluctuations around $a_{2} \simeq 0$, inspite of the large system sizes $\left(N=10^{6}\right)$ simulated here. The ICS consists of clusters of particles streaming in independent directions - an averaging over these clusters is expected to yield a Gaussian distribution of velocities [16, 18, 28]. Our late-time results are consistent with the MB result $a_{2}=0$, but the average value of $a_{2}$ still shows large variations with $\tau$.

In Fig. 6, we plot the Sonine coefficients $a_{2}, a_{3}, a_{4}, a_{5}$ vs. $\tau$ for the same parameter values as in Fig. 5. We observe that the velocity distributions in all cases are primarily described by the coefficient $a_{2}$ - the values of higher coefficients are smaller by upto an order of magnitude. In general, the behavior of the higher coefficients is analogous to that for $a_{2}$ in Fig. 5. There is an early-time regime where the data sets for independent runs are approximately coincident. At later times, there is a large variation in the $a_{n}$-values for various runs - the corresponding averages are shown in Fig. 6. 
(a) $d=2, e=0.8$

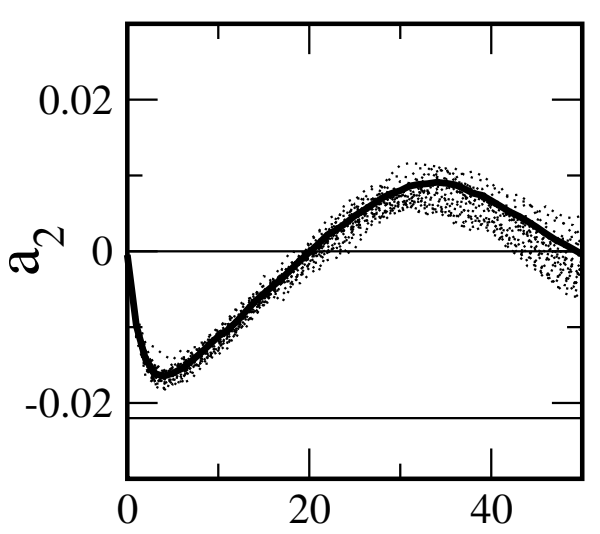

(c) $d=3, e=0.9$

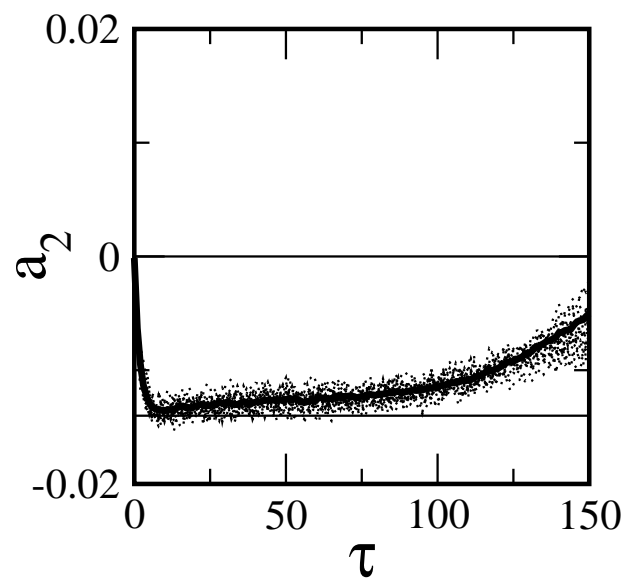

(b) $\mathrm{d}=2, \mathrm{e}=0.8$

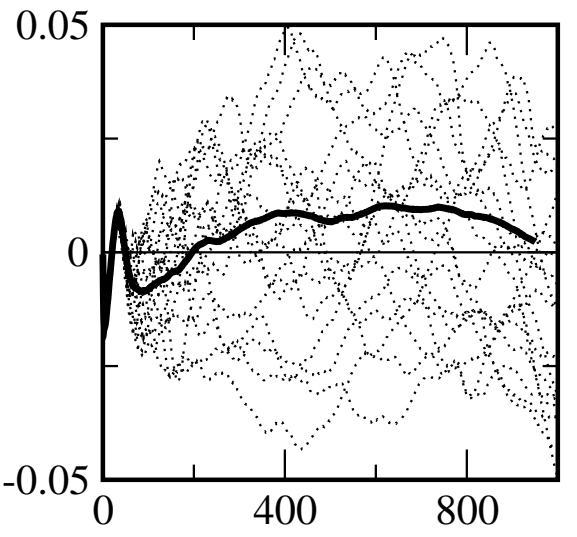

(d) $d=3, e=0.9$

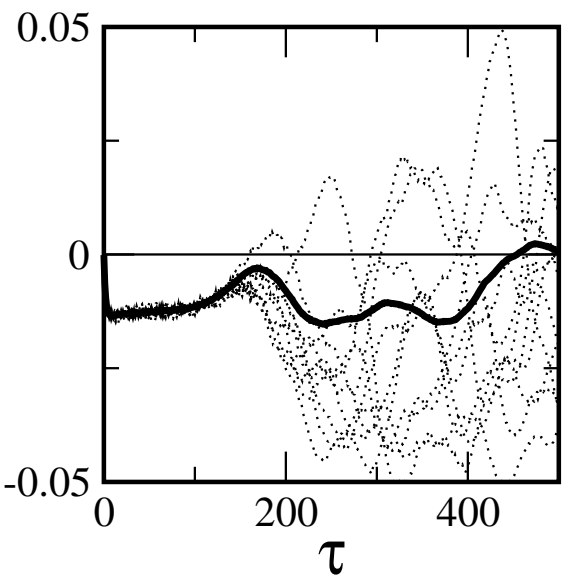

Figure 5: (a)-(b) Time-dependence of $a_{2}$ for $d=2, e=0.8$. The dotted lines denote $a_{2}$ vs. $\tau$ for individual runs, and the solid line is the corresponding average. The frames on the left and right show the early-time and late-time behaviors, respectively. A horizontal line is drawn in (a) at $a_{2} \simeq-0.022$, corresponding to the kinetic-theory prediction in Eq. (2.13) [21]. The HCS $\rightarrow$ ICS crossover occurs at $\tau_{c} \simeq 48$. (c)-(d) Analogous to (a)-(b) but for $d=3, e=0.9$. The line in (c) corresponds to $a_{2} \simeq-0.015$. The HCS $\rightarrow \mathrm{ICS}$ crossover is $\tau_{c} \simeq 212$.

\section{Summary and Discussion}

Let us conclude this paper with a summary and discussion of the results presented here. We have undertaken large-scale molecular dynamics (MD) simulations of freely-evolving granular gases. The system initially loses energy (or cools) in a homogeneous cooling state (HCS). At later times $\left(\tau \gg \tau_{c}\right.$, where $\tau_{c}$ is a crossover time), the growth of fluctuations in the density and velocity fields drives the system into an inhomogeneous cooling state (ICS). Our primary focus in this paper is the nature of velocity distributions in the HCS and ICS. We model the velocity distributions by a Sonine polynomial expansion [cf. Eq. (2.10)]. The magnitude of the Sonine coefficients $a_{n}$ measures the departure from the Maxwell-Boltzmann (MB) distribution function.

We find that the velocity distribution in all cases is primarily described by the coefficient $a_{2}$. In 
(a) $\mathrm{d}=2, \mathrm{e}=0.8$

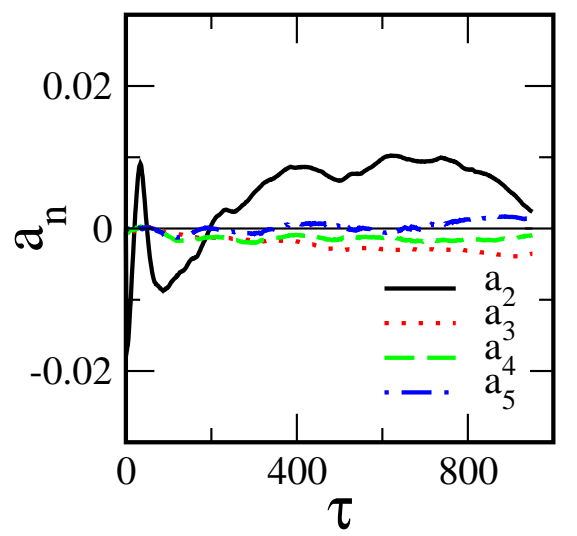

(b) $d=3, e=0.9$

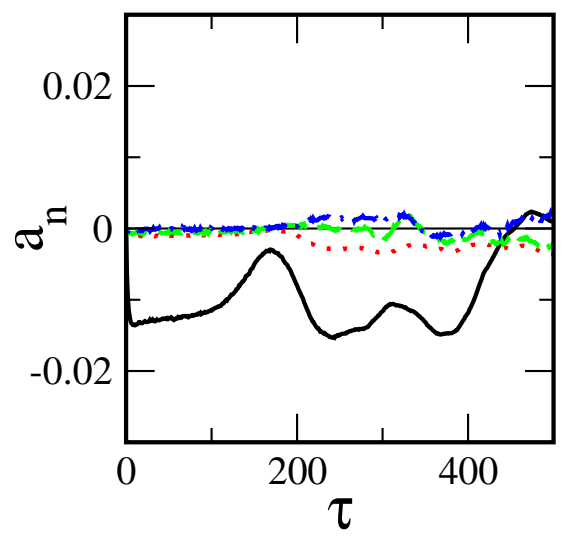

Figure 6: Time-dependence of Sonine coefficients $a_{2}, a_{3}, a_{4}, a_{5}$ for (a) $d=2, e=0.8$; (b) $d=3, e=0.9$.

the HCS, $a_{2}$ saturates to a value which is consistent with the kinetic-theory prediction in Eq. (2.13). However, at later times, our MD results for $a_{2}$ are consistent with the MB value $a_{2}=0$. We should stress that individual data sets for $a_{2}$ vs. $\tau$ show strong fluctuations around $a_{2}=0$, inspite of the large system sizes $\left(N=10^{6}\right)$ and averaging (50 initial conditions) in our MD simulations.

Our present interests in this problem include the aging behavior of the velocity autocorrelation function during the cooling process. We will present results on this in an extended publication [29].

Acknowledgments: SP is grateful to S. Luding, I. Goldhirsch, H. Herrmann, H. Nakanishi, and M. Ernst for useful discussions on the problems discussed in this paper. He is also grateful to the Department of Science and Technology for supporting this research through a project on Pattern Formation in Granular Materials. SRA wishes to thank CSIR, India for financial support.

\section{References}

[1] H.M. Jaeger, S.R. Nagel, and R.P. Behringer, Rev. Mod. Phys. 68, 1259 (1996).

[2] Powders and Grains 97: Proceedings of the Third International Conference on Powders and Grains, ed. R.P. Behringer and J. Jenkins (Balkema, Rotterdam, 1997).

[3] Powders and Grains 2001: Proceedings of the Fourth International Conference on Powders and Grains, ed. T. Kishino (Swets and Zeitlinger, Lisse, 2001).

[4] G.H. Ristow, Pattern Formation in Granular Materials (Springer-Verlag, Berlin, 2000).

[5] J. Duran, Sands, Powders and Grains (Springer-Verlag, Berlin, 2000).

[6] N.V. Brilliantov and T. Poschel, Kinetic Theory of Granular Gases (Oxford University Press, Oxford, 2004).

[7] J. Rajchenbach, Phys. Rev. Lett. 65, 2221 (1990); S. Das Gupta, D.V. Khakhar and S.K. Bhatia, Chem. Eng. Sci. 46, 1531 (1991); O. Zik, D. Levine, S.G. Lipson, S. Shtrikman and J. Stavans, Phys. Rev. Lett. 73, 644 (1994).

[8] S. Puri and H. Hayakawa, Physica A 270, 115 (1999); S. Puri and H. Hayakawa, Physica A 290, 218 (2001). 
[9] F. Melo, P.B. Umbanhowar and H.L. Swinney, Phys. Rev. Lett. 75, 3838 (1995); P.B. Umbanhowar, F. Melo and H.L. Swinney, Nature 382, 793 (1996).

[10] P.K. Haff, J. Fluid Mech. 134, 401 (1983).

[11] I. Goldhirsch and G. Zanetti, Phys. Rev. Lett. 70, 1619 (1993); I. Goldhirsch, M.-L. Tan and G. Zanetti, J. Sci. Comput. 8, 1 (1993).

[12] S. McNamara and W.R. Young, Phys. Fluids A 4, 496 (1992); Phys. Rev. E 53, 5089 (1996).

[13] J.J. Brey, F. Moreno and J.W. Dufty, Phys. Rev. E 54, 445 (1996); J.J. Brey, F. Moreno and M.J. Ruiz-Montero, Phys. Fluids 10, 2965 (1998); Phys. Fluids 102976 (1998).

[14] T.P.C. van Noije, M.H. Ernst, R. Brito and J.A.G. Orza, Phys. Rev. Lett. 79, 411 (1997); T.P.C. van Noije, M.H. Ernst and R. Brito, Phys. Rev. E 57, R4891 (1998); T.P.C. van Noije and M.H. Ernst, Phys. Rev. E 61, 1765 (2000).

[15] S. Luding, M. Huthmann, S. McNamara, and A. Zippelius, Phys. Rev. E 58, 3416 (1998); S. Luding and S. McNamara, Granular Matter 1, 113 (1998); S. Luding and H.J. Herrmann, Chaos 9, 673 (1999).

[16] A. Baldassarri, U.M.-B. Marconi and A. Puglisi, Phys. Rev. E 65, 051301 (2002); U.M.-B. Marconi, A. Puglisi and A. Baldassarri, Phase Transitions 77, 863 (2004).

[17] S.K. Das and S. Puri, Europhys. Lett. 61, 749 (2003); S.K. Das and S. Puri, Phys. Rev. E 68, 011302 (2003).

[18] H. Nakanishi, Phys. Rev. E 67, R010301 (2003).

[19] S. Chapman and T.G. Cowling, The Mathematical Theory of Non-Uniform Gases (Cambridge University Press, New York, 1970).

[20] A. Goldstein and M. Shapiro, J. Fluid Mech. 282, 75 (1995).

[21] T.P.C. van Noije and M.H. Ernst, Granular Matter 1, 57 (1998).

[22] J.J. Brey, D. Cubero and M.J. Ruiz-Montero, Phys. Rev. E 54, 3664 (1996).

[23] M. Huthmann, J.A.G. Orza and R. Brito, Granular Matter 2, 189 (2000).

[24] M.P. Allen and D.J. Tildesley, Computer Simulation of Liquids (Oxford University Press, Oxford, 1987).

[25] D.C. Rapaport, The Art of Molecular Dynamics Simulation, Second edition (Cambridge University Press, Cambridge, 2005).

[26] A.J. Bray and S. Puri, Phys. Rev. Lett. 67, 2670 (1991); S. Puri, Phys. Lett. A 164, 211 (1992).

[27] J. Wakou, R. Brito and M.H. Ernst, J. Stat. Phys. 3, 107 (2002).

[28] X. Nie, E. Ben-Naim and S. Chen, Phys. Rev. Lett. 89, 204301 (2002).

[29] S.R. Ahmad and S. Puri, in preparation. 